\title{
The effects of Le Fort I osteotomy on velopharyngeal function in cleft patients
}

\author{
Suvi Alaluusua a, *, Leena Turunen ${ }^{a}$, Anne Saarikko a, Ahmed Geneid ${ }^{\text {b }}$, Junnu Leikola a, \\ Arja Heliövaara ${ }^{a}$ \\ ${ }^{a}$ Cleft Palate and Craniofacial Center, Department of Plastic Surgery, (Head of Department Erkki Tukiainen, Professor, MD, PhD), Helsinki University \\ Hospital, P.O. Box 266, FI-00029, HUS, Finland \\ b Department of Otorhinolaryngology and Phoniatrics, Head and Neck Surgery, (Head of Department Antti Aarnisalo, Adjunct Professor, MD, PhD), Helsinki \\ University Hospital, P.O. Box 220, FI-00029, HUS, Finland
}

\section{A R T I C L E I N F O}

\section{Article history:}

Paper received 15 August 2018

Accepted 16 November 2018

Available online $\mathrm{xxx}$

\section{Keywords:}

Cleft palate

Maxillary advancement

Velopharyngeal function

Osteotomy

Speech

\begin{abstract}
A B S T R A C T
Introduction: Maxillary advancement may affect speech in cleft patients. The aim of this study was to evaluate whether preoperative velopharyngeal (VP) function and cleft type can predict VP function after a Le Fort I maxillary osteotomy.

Materials and methods: One hundred consecutive nonsyndromic cleft patients (54 females, 64 males) who underwent Le Fort I osteotomies were retrospectively evaluated. Pre- and postoperative VP function was assessed perceptually and instrumentally by a Nasometer. A five-point scale was used to rate velopharyngeal insufficiency symptoms (VPI $0-4$ ). To assess reliability, 30 video recordings were reevaluated.

Results: Preoperatively, $89 \%$ of patients had normal or insignificant VPI (0-1), and only $3 \%$ had moderate VPI (3). Postoperatively, $77 \%$ of patients had VPI values of $0-1$ and $14 \%$ had moderate to severe VPI values (VPI 3-4). A positive correlation was found between pre- and postoperative VPI scores, whereas the cleft type did not affect speech results. Patients with a preoperatively normal VPI (0) were not at risk for postoperative velopharyngeal incompetence.

Conclusions: There was an overall significant negative change in speech after a Le Fort I osteotomy. Atrisk patients presented with borderline (1) or more severe VPI (2 and 3) preoperatively.
\end{abstract}

(c) 2018 European Association for Cranio-Maxillo-Facial Surgery. Published by Elsevier Ltd. All rights reserved.

\section{Introduction}

Maxillary retrusion is a common problem in patients with a cleft lip and palate (Semb and Shaw, 1996). The Le Fort I osteotomy is commonly used for the correction of maxillary deformities in cleft and noncleft patients. The need for a Le Fort I osteotomy varies according to the cleft type. In recent literature, the lowest frequency found for a Le Fort I osteotomy was 13\% and was performed for an isolated cleft palate (CP) (Antonarakis et al., 2015), whereas patients with a unilateral cleft lip and palate (UCLP) or a bilateral cleft lip and palate (BCLP) exhibited higher frequencies of 40\%-76.5\% (Daskalogiannakis and Mehta, 2009; Heliövaara and Rautio, 2011; Heliövaara et al., 2013).

\footnotetext{
* Corresponding author. Cleft Palate and Craniofacial Center, Department of Plastic Surgery, Helsinki University Hospital, P.O. Box 266, FI-00029, HUS, Finland. Fax: +358 947187654

E-mail address: suvi.alaluusua@hus.fi (S. Alaluusua).
}

Orthognathic surgical treatment aims to correct maxillary hypoplasia and dental occlusion as well as to improve the patient's dentofacial appearance and self-esteem. The improvement to dental occlusion due to maxillary advancement may improve articulation (Schwarz and Gruner, 1976; Witzel et al., 1980; Ruscello et al., 1986) and have a beneficial effect on nasal patency for breathing. The improvement in nasal airway patency may contribute to the elimination of previously existing hyponasality that is secondary to the impairment of the nasal cavities (Warren and Drake, 1993; Trindade et al., 2003).

However, when the maxilla is advanced surgically, the hard palate and the attached soft palate are advanced as well, which can affect velopharyngeal function. According to several studies, correcting maxillary retrusion may have a negative effect on cleft patients' speech (Witzel and Munro, 1977; Epker and Wolford, 1976; Watzke et al., 1990; Haapanen et al., 1997; Maegawa et al., 1998; Trindade et al., 2003; Heliövaara et al., 2004; Janulewicz et al., 2004; Niemeyer et al., 2005; Pereira et al., 2013) and trigger 
or worsen velopharyngeal incompetence and/or insufficiency (VPI). VPI is defined as a dysfunction of the velum and/or the pharyngeal wall at the level of the nasopharynx that results in inadequate velopharyngeal closure (Peterson-Falzone et al., 2010). VPI is a typical problem among cleft patients and causes speech symptoms such as hypernasality, nasal emissions, and weak pressure consonants. As a result, speech intelligibility may be reduced.

However, the reported effects of maxillary advancement on velopharyngeal function and speech are still quite contradictory, especially in cleft patients. In a review article by Chanchareonsook et al. (2006), the results of studies about the effect of maxillary advancement on velopharyngeal function in cleft patients are divided into three categories: (a) no relationship between maxillary advancement and velopharyngeal status, (b) deterioration of velopharyngeal status after maxillary advancement, and (c) deterioration of velopharyngeal status after maxillary advancement in cases with preoperative borderline velopharyngeal status. The borderline VP competence has been defined by Witzel (1989) as follows: "Borderline VP function is usually seen in the patients who exhibit a small pinhole gap in the velopharyngeal valve at maximum closure but present with normal resonance or clinically insignificant hypernasality and inaudible nasal emissions."

Several methods have been used to analyse speech before and after maxillary advancement. These have included both perceptual and instrumental methods such as nasopharyngoscopy, nasometry, videofluoroscopy and nasal airflow studies. Most of the methods (74\%) used have been perceptual (Chanchareonsook et al., 2006). According to Pereira et al., 2013 the evidence on velopharyngeal function is mixed and conflicting and seems to vary depending on which instrumental method is used. Another reason for the results being inconclusive are the small sample sizes.

Previous studies on the relationship between maxillary advancement and VPI have rarely considered whether cleft type affects the potential deterioration of velopharyngeal competence.

The aim of this study was to evaluate whether preoperative velopharyngeal function and cleft type can predict postoperative velopharyngeal function after a Le Fort I maxillary osteotomy. The hypothesis was that the preoperative symptoms of velopharyngeal incompetence (VPI) and cleft type negatively influence the postoperative velopharyngeal function, especially in those patients with uni- and bilateral cleft lip and palate and borderline velopharyngeal function.

\section{Materials and methods}

This retrospective study focused on 100 Caucasian patients with nonsyndromic cleft lip and palate or with cleft palate who underwent a Le Fort I maxillary advancement to correct midfacial retrusion at the Cleft Palate and Craniofacial Center (Cleft Center), Department of Plastic Surgery, Helsinki University Central Hospital between 2006 and 2016. Patients were operated on by two experienced high-volume cleft surgeons (Board certified, over 10 years of experience, more than 30 operations per year, head Professor Erkki Tukiainen). The osteotomies were grafted using bone from the iliac crest and were fixed with titanium plates. Three patients underwent an early osteotomy during growth, and 27 patients

Table 1

Sex and type of cleft among patients with a Le Fort 1 osteotomy $(\mathrm{n}=100)$.

\begin{tabular}{llll}
\hline & Boys & Girls & Total \\
\hline Cleft palate (CP and SMCP) & 6 & 17 & 23 \\
Unilateral cleft lip and palate (UCLP) & 28 & 25 & 53 \\
Bilateral cleft lip and palate (BCLP) & 13 & 11 & 24 \\
Total & 47 & 53 & 100 \\
\hline
\end{tabular}

underwent bimaxillary osteotomies. The sample consisted of 54 female and 46 male patients (Table 1). Patients' ages ranged from 11.5 to 45.3 years, with a mean of 17.9 years (SD 4.2 ). The cleft type distribution was as follows: 23 patients had palatal clefts (20 isolated cleft palate (CP) and 3 submucous palate (SMCP) and 77 had clefts of the lip and palate (53 UCLP and 24 BCLP). Two of the submucous clefts ere operated and 1 was not. We excluded patients with the following characteristics: distraction was used to advance the maxilla, the postoperative evaluation was missing, the cleft was part of a syndrome, the patient had severe dyspraxia, patient was not able to read or had a facial cleft. The patients included in the study all spoke Finnish as their mother tongue.

In total, 31 patients (9 CP, 13 UCLP, $9 \mathrm{BCLP}$ ) had undergone corrective speech surgery before undergoing a Le Fort I osteotomy. Among these, 19 patients (6 CP, 7 UCLP, 6 BCLP) had a velopharyngeal flap. Six patients (2 CP, 3 UCLP, 1 BCLP) underwent Furlow's repalatoplasty and 6 (1 CP, 3 UCLP, 2 BCLP) underwent two speech corrective surgeries, Furlow's repalatoplasty and a velopharyngeal flap.

The study protocol was approved by Helsinki University Central Hospital ( $\S 27 / 2015$, Nina Lindfors, Adjunctive professor, Principal Investigator). The study did not fulfill characteristics of a medical study according to the Medical Research Act and did not need an ethical permission. Principles outlined in the Declaration of Helsinki were followed.

\subsection{Assessment of speech and VP function}

To assess the VP function, speech data were reviewed from hospital records. Pre- and postoperative VP function had been assessed perceptually and instrumentally with a Nasometer (Kay Pentax Nasometer II, model 6400, Kay Elemetrics, 2001). The postoperative speech evaluation took place 6-12 months (mean 8.7 months, SD 4.4) after the Le Fort I osteotomy. Evaluations were performed by six Board certified speech pathologists who all had over 2 years of experience (range 2-30 years) in evaluating cleft speech characteristics. All speech pathologists were working full time at the Cleft Palate and Craniofacial Center and evaluating more than 500 patients per year.

Hypernasality, nasal emissions, and compensatory articulation or weakness of the pressure consonants/p, t, k/were perceptually rated and coded according to the speech evaluation form routinely used at the Cleft Center to evaluate VPI features. To rate VPI symptoms, a five-point scale was used, and the degree of velopharyngeal insufficiency was assessed as follows: $0=$ normal velopharyngeal competence, 1 = insignificant, borderline, mild and occasional VPI detected by ears and/or nasometer, $2=$ mild and consistent VPI, $3=$ moderate and consistent VPI, $4=$ severe and consistent VPI. For insignificant VPI (1), nasality or nasal emissions were detected only temporarily, and the speech sound was normal or nearly normal. In addition, pressure consonants were not affected, and no speech correction treatment was required. With mild VPI (2), speech was mildly nasal at all times and pressure consonants were not affected. A moderate VPI (3) indicated that the subjects' speech featured moderate nasality at all times and a weakness in pressure consonants was detected. In the case of severe VPI (4), speech was highly nasal and pressure consonants were

Table 2

A five-point scale to assess the degree of velopharyngeal insufficiency (VPI).

\begin{tabular}{ll}
\hline Normal velopharyngeal competence & 0 \\
Insignificant, borderline VPI & 1 \\
Mild and consistent VPI & 2 \\
Moderate and consistent VPI & 3 \\
Severe and consistent VPI & 4 \\
\hline
\end{tabular}


either weak or replaced by glottal stops. In some cases of mild, moderate and severe VPI, nasal emissions were present (Table 2).

Nasality was evaluated perceptually but also confirmed instrumentally with the Nasometer, which served to validate the perceptual assessment. As a reference scale, we used a five-point scale that was created for young Finnish cleft adults (Haapanen, 1991). Values higher than 30\% (scale value 2) were considered to be a symptom of VPI (Smedberg et al., 2014). In the speech analysis, the Finnish naming test originally designed for the Scandcleft project (Lohmander et al., 2009) was used. In total, 30 target words and 7 sentences were originally selected for the test. In our study, we used only plosives/p, $\mathrm{t}, \mathrm{k} /$ because of their particular vulnerability to the VPI condition. The words included six target words each for the plosives/p, $\mathrm{t}, \mathrm{k} /$. The chosen consonants/p t k/occurred in each word in the initial position of the stressed syllable followed by a vowel. For some words, the test consonant was followed by a high vowel intended for the assessment of hypernasality.

Patients read 30 separate words and 7 sentences. The sentences used in the perceptual analysis were identical to those used in the nasometry evaluation. Seven sentences consisted of only oral consonants (/Atte ottaa tuttia/,/papa puhuu puppua/,/kuka tekee papupataa/,/viivi heijaa vauvaa/,/olli laulaa lailaa/,/assi sössöttää/,/harri pärrää/). One extra sentence (/minne mummo meni/) consisted of nasal consonants but was not used in our study because we did not evaluate hyponasality. The text(Vaahteranmäen Eemeli) is routinely used to document connected speech at our center and was used to evaluate connected speech in these speech evaluations as well. This text contains 61 words and takes approximately $30 \mathrm{~s}$ to read.

To increase reliability, 30 pre- and postoperative video recordings were re-evaluated by two cleft-experienced speech therapists. Intra- and inter-rater agreement was calculated for the completed evaluations. The assessment was done separately in a quiet room where the therapists listened through high-quality headphones (Creative Aurvana Live, Creative Technology Ltd, Singapore).

\subsection{Statistical analyses}

Pre- and postoperative speech changes were tested statistically using the sign-test and Spearman's correlation. Kappa statistics were calculated to assess reliability. Chi-square was used to assess the effect of the cleft type on speech changes. A statistician (who

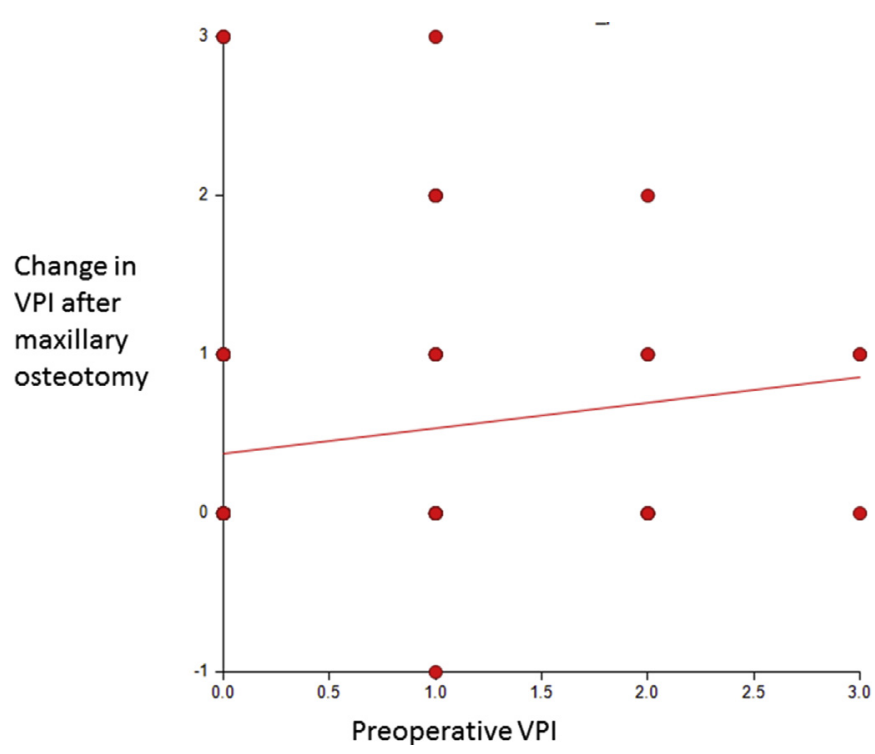

Fig. 1. Positive correlation of pre- and postoperative VPI. was not involved in the study) calculated the analyses. The patients were analysed anonymously.

\section{Results}

Using the sign-test, we found an overall negative change in speech following the Le Fort I osteotomy $(P<0.001)$. We also found a positive correlation between the pre- and postoperative VPI scores (Fig. 1). The cleft type did not affect the speech results $(0.745, \mathrm{~ns})$.

Preoperatively, $89 \%$ of patients had a normal or insignificant VPI (0-1), and only $3 \%$ had a moderate VPI (3). Postoperatively, $77 \%$ of patients had VPI values of $0-1$ and $14 \%$ had moderate to severe VPI (VPI 3-4). Pre- and postoperative values of VPI in all cleft patients and values of VPI by cleft type are shown in Figs. 2-5, dark green referring to normal velopharyngeal competence (VPI 0), light green to insignificant (VPI 1), yellow to mild and consistent (VPI 2), orange to moderate (VPI 3) and red to severe (VPI 4).

In total, 14 patients ( $2 \mathrm{CP}, 6 \mathrm{UCLP}$, and $6 \mathrm{BCLP})$ required corrective speech surgery; however, one patient declined surgery because she was happy with her speech. Patients with preoperatively normal velopharyngeal function, VPI $(0)$, were not at risk for postoperative velopharyngeal incompetence. However, in 16 patients with preoperatively normal VP function, VPI (0), insignificant VPI (1) was noticed postoperatively. Furthermore, in 3 patients the pharyngeal flap was resected during osteotomy, and increased VPI values (from 0 to 3 ) were recorded postoperatively. In total, 31 patients had undergone corrective speech surgery before osteotomy. In 19 of these patients, pharyngeal flaps were used.

The perceptual re-evaluations completed by two speech pathologists were compared against each other, and the intra- and interrater reliabilities were calculated and found to vary between

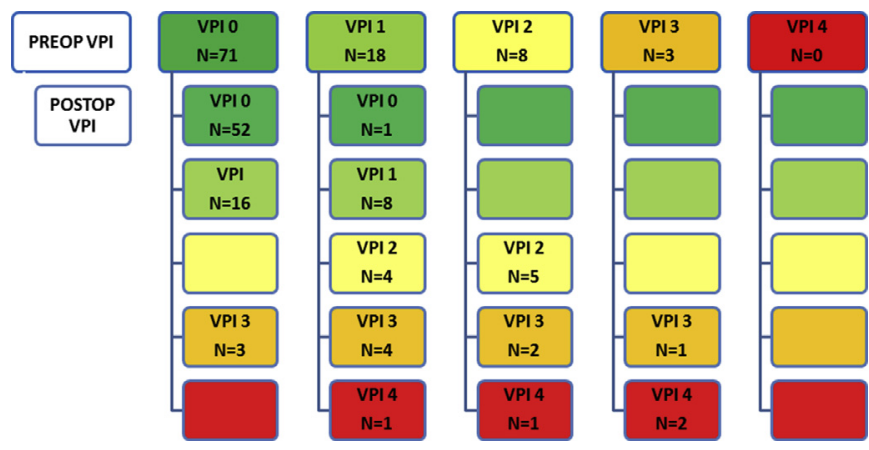

Fig. 2. Pre- and postoperative VPI values in 100 cleft patients ( 53 UCLP, 24 BLCP, 20 CP and $3 \mathrm{SMCP}$ ). Dark green referring to normal velopharyngeal competence (VPI 0), light green to insignificant (VPI 1), yellow to mild and consistent (VPI 2), orange to moderate (VPI 3) and red to severe (VPI 4).

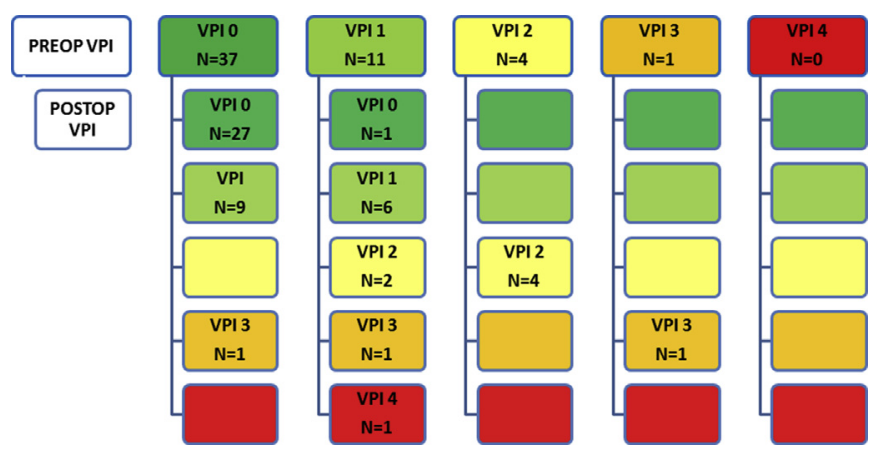

Fig. 3. Pre-and postoperative values of VPI in 53 patients with UCLP. 


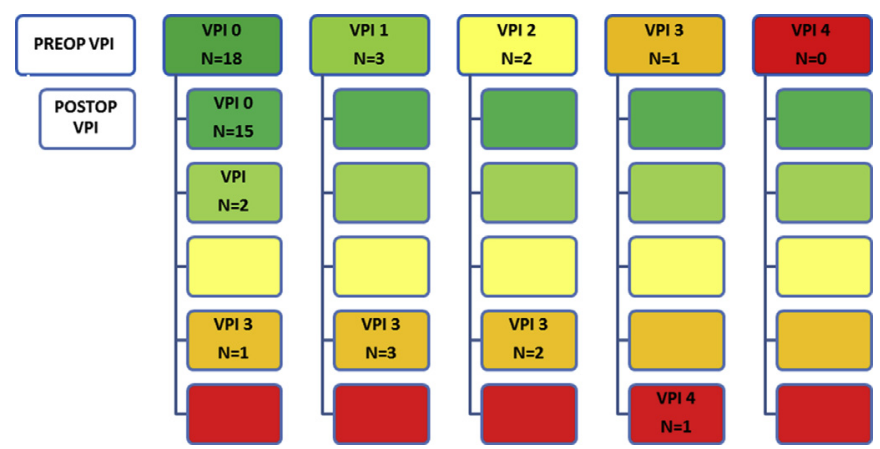

Fig. 4. Pre-and postoperative values of VPI in 24 patients with BCLP.

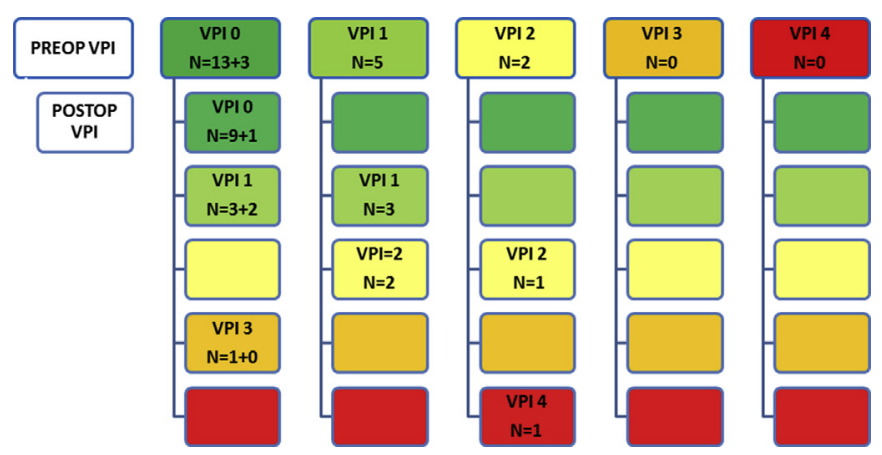

Fig. 5. Pre-and postoperative values of VPI in 23 patients with $\mathrm{CP}(20 \mathrm{CP}$ and $3 \mathrm{SMCP})$.

good and excellent agreement (0.728-0.959 and 0.622-0.842, respectively).

\section{Discussion}

\subsection{Preoperative VPI}

Our results indicate that there was an overall negative change in speech after the Le Fort I osteotomy. Several studies using different methods have agreed with our findings and have concluded that there is a deterioration of velopharyngeal status after maxillary advancement (Watzke et al., 1990; Haapanen et al., 1997; Trindade et al., 2003; Heliövaara et al., 2004; Pereira et al., 2013). Watzke et al. (1990) studied alterations in velopharyngeal function after maxillary advancement in 24 cleft patients (4 BCLP, 20 UCLP) using aerodynamic assessment. They found impaired VP function in five patients but also improvement in five patients. Trindade et al. (2003) evaluated 29 cleft patients (4 CP, 18 UCLP, 7 BCLP) using aerodynamic assessment and a Nasometer. A significant increase in mean nasalance score was found. Haapanen et al. (1997) also reported a significant deterioration of VP function based on perceptual speech assessment in $27 \%$ of 15 investigated cleft patients (10 UCLP, 5 BCLP) but found no change in postoperative aerodynamic data or results from nasometry. Heliövaara et al. (2004) used composite scores for perceptual evaluations of 50 cleft patients (30 UCLP, 9 BCLP, 11CP). They included parameters similar to our parameters. The degree of VPI was based on the severity of the VPI symptoms.

According to our study, the risk of developing significant VPI was higher among patients exhibiting VPI symptoms before osteotomy (VPI 1-3). This is in agreement with the work of Witzel (1989), which states that preoperative borderline velopharyngeal status is a potential risk factor in the context of maxillary advancement. They evaluated 41 patients without cleft palate and 50 patients with cleft palate or cleft lip and palate who underwent Le Fort I maxillary advancement. Eleven of the 15 study patients who had a repaired cleft palate and a preoperative rating of borderline VP closure acquired surgically induced symptomatic VPI after Le Fort I advancement. For most patients, the extent of preoperative competence of the VP sphincter before Le Fort I advancement predicted the competence of the VP sphincter after surgery as documented by videofluoroscopy and nasoendoscopy. Janulewicz et al. (2004) had similar results. They evaluated VP function perceptually using Pittsburg-weighted values for speech symptoms associated with velopharyngeal incompetence in 54 cleft patients (28 UCLP, 21 BCLP, 4 CP) who underwent maxillary Le Fort I advancement. Their conclusion was that patients with borderline preoperative function are at risk for VPI after maxillary advancement.

Several studies have shown, however, that maxillary advancement does not deteriorate velopharyngeal status (Schwarz and Gruner, 1976; Kummer et al., 1989; Maegawa et al., 1998; and Smedberg et al., 2014). Smedberg et al. (2014) investigated the impact of maxillary advancement on velopharyngeal function and symptoms of VPI in 13 cleft patients (8 BCLP, 5 UCLP) using perceptual, acoustic (Nasometer) and visual (Videofluoroscopy and Videonasoendoscopy) evaluation. They found no evidence of significant impact on the VP function at the group level, but three individuals had a somewhat deteriorated VP function postoperatively. Kummer et al. (1989) made similar conclusions with a perceptual and multiview videofluoroscopic speech study. Two patient groups were evaluated: 8 cleft patients ( $7 \mathrm{CLP}, 1 \mathrm{CL}$ ) and 8 patients without a cleft. The authors found no increased risk for postoperative hypernasality among patients with or without a cleft. In both studies, there were individual changes, but these changes were not considered to be clinically significant. The sample sizes in these studies were rather small.

\subsection{Cleft type}

We expected that cleft type would negatively influence the postoperative velopharyngeal function, especially in those patients with uni- and bilateral cleft lip and palate and borderline velopharyngeal function. Interestingly, the cleft type did not affect the VPI features, although the need for a Le Fort I osteotomy is higher for a cleft lip and palate than for an isolated cleft palate (Antonarakis et al., 2015; Daskalogiannakis and Mehta, 2009). Existing literature on this subject remains scarce. Information concerning the relationship between the deterioration of velopharyngeal status after maxillary advancement and the cleft type requires a large number of subjects, and, so far, most of the studies have had quite small sample sizes (Chanchareonsook et al., 2006; Pereira et al., 2013). The compensatory ability of a cleft patient following a maxillary osteotomy can be impaired due to issues such as scarring, a short soft and hard palate, improper muscular anatomy, and an already extended compensatory mechanism. In addition, the amount of maxillary advancement may affect velopharyngeal functioning. Unfortunately, we did not study the amount of maxillary horizontal and vertical movement. It may be that patients with more severe maxillary growth disturbance require larger maxillary advancements, potentially further affecting speech.

\subsection{Pre-existing pharyngeal flap}

Pharyngeal flaps are effective in eliminating hypernasality secondary to primary palatoplasty, eliminating as much as $88 \%$ of VPI symptoms (Ysunza et al., 2002). For cleft patients requiring orthognathic surgery, the pre-existing velopharyngeal flap may present a problem. In our study, 19 patients had a pre-existing velopharyngeal flap preoperatively. Three cases involved the resection of a preexisting pharyngeal flap during osteotomy. All of these cases 
deteriorated from normal velopharyngeal competence (VPI 0 ) to moderate VPI (VPI 3). Although our sample was too small for statistical analysis, resection of the flap may represent a risk factor for the development of significant postoperative VPI. In addition, a preexisting flap may increase postoperative relapse (Haapanen et al., 1997; Posnick et al., 1990) since it may decrease one's ability to mobilize and stabilize an advanced maxilla. Among CP patients, $45 \%$ $(9 / 20)$ required VPI surgery before osteotomy following a primary cleft procedure, a significantly higher proportion than patients with other cleft types. On the other hand, Watzke et al. (1990) found improvement in 5 of 24 patients after maxillary advancement, and these five patients had a pharyngeal flap in place at surgery.

\subsection{Methodology}

According to Pereira et al. (2013), the gold standard for evaluating velopharyngeal function would be perceptual speech analysis, acoustic measurement with Nasometer and visual perceptual assessment with videofluorography (VF) and videonasoendoscopy (VNE). Smedberg et al. (2014) concluded that nasoendoscopy of VF should be included in patients with syndromes and/or additional malformations but, with a typical cleft patient, perceptual speech evaluation and nasometry would be sufficient. In addition, according to a study by Phillips et al. (2005), nasoendoscopy did not significantly contribute to the information obtained from perceptual speech assessment.

Our assessment relied on perceptual evaluation and Nasometer tests to evaluate typical VPI features. Nasalance scores appear to correlate with the perceptual judgment of hypernasality rated by trained listeners (Dalston et al., 1991) and, as such, may provide an objective measurement strong enough for evaluation. With the combination of perceptual evaluation and nasometry, we were able to predict the patients who were at risk of developing significant VPI. Slight nasality was sometimes detected and other times seen only in the nasometry. With perceptual evaluation, only borderline VPI was not always noted, despite the listeners' experience evaluating cleft speech characteristics. Based on our study, it is important to use both perceptual and instrumental evaluation to predict if a patient is at risk of developing significant VPI after maxillary advancement. Chanchareonsook et al. (2006) pointed out that most studies on maxillary advancement and speech have used perceptual analysis alone. This might partially explain why results are controversial.

A strength of our study is the large sample size of cleft patients. Reviews by Chanchareonsook et al. (2006) and Pereira et al. (2013) recognized the need for sufficient sample sizes to understand the impact of maxillary advancement on VPI and other speech characteristics. The same review articles have also pinpointed the need for inter- and intra-rater reliability tests. In our study, inter- and intrarater analyses indicated a high degree of agreement and reliability.

The timing of postoperative evaluation seems to be critical. Trindade et al. (2003) found transient deterioration in nasalance approximately 45 days after the maxillary advancement and noted that the increase in nasalance scores was much less dramatic at 6 months postoperative evaluation. According to Pereira et al. (2013), the deterioration seen at 3 months after maxillary advancement was stable. We evaluated speech 6-12 months after Le Fort I.

\subsection{Clinical significance}

The speech of cleft patients needs to be considered carefully when planning maxillary advancement. Informing patients with preoperative borderline VPI about the risks and the potential need for a secondary corrective speech surgery after maxillary advancements remains essential. Based on our study, maxillary advancement appears to be a safe operation for patients who have a normal velopharyngeal competence preoperatively. The velopharyngeal function in this population of cleft patients can deteriorate but not in a clinically significant manner. According to previous studies, maxillary advancement may also result in positive changes in speech articulation, which is also important to recognize.

\section{Conclusions}

The results of this study suggest that maxillary advancement using a Le Fort I osteotomy increases VPI features in cleft patients. Patients exhibiting normal preoperative resonance are not at risk of developing significant VPI. However, patients presenting with borderline VPI are at risk of developing significant VPI, regardless of the type of cleft.

\section{Funding}

Authors report no sources of support.

\section{References}

Antonarakis GS, Watts G, Daskalogiannakis J: The need for orthognathic surgery in nonsyndromic patients with repaired isolated cleft palate. Cleft Palate Craniofac J 52: 8-13, 2015

Chanchareonsook N, Samman N, Whitehill TL: The effect of cranio-maxillofacial osteotomies and distraction osteogenesis on speech and velopharyngeal status: a critical review. Cleft Palate Craniofac J 43: 477-487, 2006

Dalston RM, Warren DW, Dalston ET: Use of nasometry as a diagnostic tool for identifying patients with velopharyngeal impairment. Cleft Palate Craniofac J 28: $184-188,1991$

Daskalogiannakis J, Mehta M: The need for orthognathic surgery in patients with unilateral and complete bilateral cleft lip and palate. Cleft Palate Craniofac J 46: 498-502, 2009

Epker BN, Wolford LM: Middle-third facial osteotomies: their use in the correction of congenital dentofacial and craniofacial deformities. J Oral Surg 34: 324-342, 1976

Haapanen ML: Nasalance scores in normal Finnish speech. Folia Phoniatr 43: 197-203, 1991

Haapanen ML, Kalland M, Heliövaara A, Hukki J, Ranta R: Velopharyngeal function in cleft patients undergoing maxillary advancement. Folia Phoniatr Logop 49: 42-47, 1997

Heliövaara A, Hukki J, Ranta R, Haapanen ML: Cephalometric pharyngeal changes after Le Fort I osteotomy in different types of clefts. Scand J Plast Reconstr Surg Hand Surg 38: 5-10, 2004

Heliövaara A, Leikola J, Hukki J: Craniofacial cephalometric morphology and the later need for orthognathic surgery in 6-year-old children with BCLP. Cleft Palate Craniofac J 50: 35-40, 2013

Heliövaara A, Rautio J: A comparison of craniofacial cephalometric morphology and the later need for orthognathic surgery in 6-year-old cleft children. J Craniomaxillofac Surg 39: 173-176, 2011

Janulewicz J, Costello BJ, Buckley MJ, Ford MD, Close J, Gassner R: The effects of Le Fort I osteotomies on velopharyngeal and speech functions in cleft patients. J Oral Maxillofac Surg 62: 308-314, 2004

Kummer AW, Strife JL, Grau WH, Creaghead NA, Lee L: The effects of Le Fort osteotomy with maxillary movement on articulation, resonance, and velopharyngeal function. Cleft Palate J 26: 193-199, 1989 discussion 199-200

Lohmander A, Willadsen E, Persson C, Henningsson G, Bowden M, Hutters B: Methodology for speech assess-ment in the Scandcleft project-an international randomized clinical trial on palatal surgery: experiences from a pilot study. Cleft Palate Craniofac J 46: 347-362, 2009

Maegawa J, Sells RK, David DJ: Pharyngoplasty in patients with cleft lip and palate after maxillary advancement. J Craniofac Surg 9: 330-335, 1998

Niemeyer TC, Gomes Ade O, Fukushiro AP, Genaro KF: Speech resonance in orthognathic surgery in subjects with cleft lip and palate. J Appl Oral Sci 13: 232-236, 2005

Pereira VJ, Sell D, Tuomainen J: Effect of maxillary osteotomy on speech in cleft lip and palate: perceptual outcomes of velopharyngeal function. Int J Lang Commun Disord 48: 640-650, 2013a

Pereira V, Sell D, Tuomainen J: The impact of maxillary osteotomy on speech outcomes in cleft lip and palate: an evidence-based approach to evaluating the literature. Cleft Palate Craniofac J 50: 25-39, 2013b

Peterson-Falzone SJ, Hardin-Jones MA, Karnell MP: Diagnosing and managing communication disorders in cleft palate. In: Falk K, Gower J, Vales S (eds), Cleft palate speech, 4th ed. St. Louis: Mosby Elsevier, 221-247, 2010

Phillips J, Klaiman P, Delorey R, MacDonald D: Predictors of velopharyngeal insufficiency in cleft palate orthognatic surgery. Plast Reconstr Surg 115: 681-686, 2005 
Posnick JC, Ewing MP: Skeletal stability after Le Fort I maxillary advancement in patients with unilateral cleft lip and palate. Plast Reconstr Surg 85: 706-710, 1990

Ruscello DM, Tekieli ME, Jakomis T, Cook L, Van Sickels JE: The effects of orthognathic surgery on speech production. Am J Orthod 89: 237-241, 1986

Schwarz C, Gruner E: Logopaedic findings following advancement of the maxilla. J Maxillofac Surg 4: 40-55, 1976

Semb G, Shaw WC: Facial growth in orofacial clefting disorders. In: Turvey TA, Vig KWL, Fonseca RJ (eds), Facial clefts and craniosynostosis. Principles and Management. Philadelphia: W.B. Saunders, 28-56, 1996

Smedberg E, Neovius E, Lohmander A: Impact of maxillary advancement on speech and velopharyngeal function in patients with cleft lip and palate. Cleft Palate Craniofac J 51: 334-343, 2014

Trindade IE, Yamashita RP, Suguimoto RM, Mazzottini R, Trindade Jr AS: Effect of orthognathic surgery on speech and breathing of subjects with cleft lip and palate: acoustic and aerodynamic assessment. Cleft Palate Craniofac J 40: 54-64, 2003

Warren DW, Drake AF: Cleft nose: form and function. Clin Plast Surg 20: 769-779, 1993

Watzke I, Turvey TA, Warren DW, Dalston R: Alterations in velopharyngeal function after maxillary advancement in cleft palate patients. J Oral Maxillofac Surg 48: 685-689, 1990

Witzel MA, Munro IR: Velopharyngeal insufficiency after maxillary advancement Cleft Palate J 14: 76-80, 1977

Witzel MA, Ross RB, Munro IR: Articulation before and after facial osteotomy. J Maxillofac Surg 8: 195-202, 1980

Ysunza A, Pamplona C, Ramírez E, Molina F, Mendoza M, Silva A: Velopharyngeal surgery: a prospective randomized study of pharyngeal flaps and sphincter pharyngoplasties. Plast Reconstr Surg 110: 1401-1407, 2002 